\title{
rSPSCs: A new type of pluripotent stem cells
}

\author{
Cell Research (2015) 25:889-890. doi:10.1038/cr.2015.73; published online 16 June 2015
}

Pluripotent stem cells capture the imagination since they can differentiate into all cell types in our body. Recent evidence suggests that in addition to embryonic stem cells (ESCs) and epiblast stem cells (EpiSCs), a new type of region-selective pluripotent stem cells (rsPSCs) exists, possessing unique spatial and molecular characteristics.

The isolation of ESCs from the inner cell mass of blastocyst stage embryos $[1,2]$ and their ability to differentiate into virtually any cell type changed the fields of developmental biology and regenerative medicine [3]. Further experiments demonstrated that epiblast stage embryos harbor another type of pluripotent stem cells, namely EpiSCs $[4,5]$. These cells have distinct molecular, cellular and functional characteristics that set them apart from ESCs (Figure 1). It was also suggested that these two cell types can convert into one another [6].

In a recent paper, Izpisua-Belmonte and colleagues [7] have isolated a new type of mouse EpiSCs, which are different from already established pluripotent cell types. By combining serum-free medium together with FGF2 and inhibition of WNT signaling they derived pluripotent stem cells with high colony formation capacities and a reduced tumorigenic potential (Figure 1). Their work provides an extensive characterization of these cells at the transcriptomic, epigenomic, proteomic and metabolomic levels. These analyses revealed that these cells resemble a subpopulation of cells from the posterior part of the post-implantation epiblast, prior to lineage commitment. Importantly, injection of these cells into post-implantation embryos in vitro yielded grafting, mainly in the posterior parts of the epiblast, suggesting the isolation of a new type of pluripotent stem cell named region-selective EpiSCs (rsEpiSCs). Furthermore, culturing of primate ESCs, including human ESCs, in the same medium conditions characteristic for the mouse rsEpiSCs allowed isolation of primate rsEpiSCs. Interestingly, transplantation of the primate rsEpiSCs into mouse embryos enabled incorporation and differentiation of the pluripotent cells into the three germ layers in a region-specific manner in these embryos.

This research should have profound impact on the study of pluripotent stem cells, mammalian development and even regenerative medicine. Conceptually, this work provides insight into the development of the early embryo, where subpopulations of pluripotent cells from different parts of the epiblast are suggested to acquire spatial differences prior to lineage differentiation. Apparently, the specification during mammalian embryogenesis is dictated not only by the differentiation into the different embryonic germ layers, but also by their location in the embryo. This differentiation paradigm gives cellular evidence that different components of the mature organism may originate from different areas of the epiblast. Based on this assumption, it may be possible to construct a fate map of mammalian development (including in humans), where for each region/organ in the mature organism, a cell of origin in the epiblast will be assigned. Furthermore, since EpiSCs are proposed to differentiate in a region-specific manner, perhaps each type of rsEpiSCs can differentiate with high efficiency into different sets of cell types. Thus, isolating different rsEpiSC lines from different regions of the epiblast may assist in increasing the efficiency of directed differentiation procedures.

The ability of the human rsEpiSCs to incorporate into the mouse embryo and create interspecies chimera is not trivial. Potentially, it would allow the study of early events in human development such as lineage commitment and differentiation. However, since the chimeras are produced ex vivo, they cannot be grown for a long period of time, and thus can only assist in the study of very early developmental stages. Furthermore, the level of grafting and contribution into the mouse tissues requires further analysis.

Although much work has been invested in trying to characterize the developmental stage of rsEpiSCs, it is still unclear whether they represent a true population of cells in the post-implantation embryo. Defining a set of markers which are specifically expressed in this subpopulation of cells, and providing evidence for their restricted expression in the posterior epiblast in vivo may provide proof for their presence during development.

These new pluripotent stem cells have features that make them adequate and even favorable for medical uses. One of the main safety issues in using pluripotent cells in regenerative medicine is the tendency of residual undifferentiated cells to form tumors, called teratoma, upon transplantation into the recipient body. Since rsEpiSCs produce smaller teratomas than EpiSCs, 


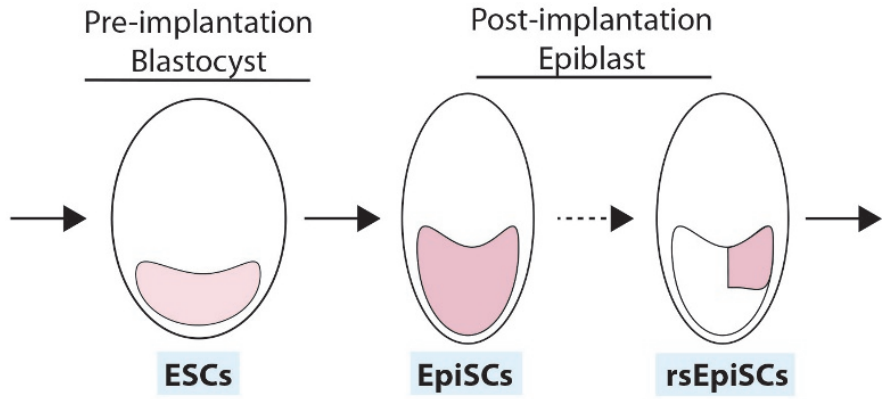

$\begin{array}{cccc}\begin{array}{c}\text { Growth } \\ \text { conditions }\end{array} & \text { 2i, LIF } & \text { FGF2, Activin-A } & \text { FGF2, WNTi } \\ \text { Clonogenicity } & \text { High } & \text { Low } & \text { High } \\ \text { Tumorigenicity } & \text { High } & \text { High } & \text { Low }\end{array}$

Figure 1 Schematic representation of early embryonic development and the pluripotent cell types isolated from each stage. As indicated, each cell type has unique characteristics related to growth conditions, clonogenicity and tumorigenicity. Continuous lines represent established differentiation pathways while dashed line represents the specification processes described in the current study.

they can be more suitable for use in regenerative medicine. In addition, due to their high cloning efficiency, rsEpiSCs show high genome editing capabilities. Furthermore, somatic cells can be reprogrammed directly into rsEpiSCs by using the unique culturing conditions of these cells. Future analyses of the differentiation capabilities and the tumorigenicity of human rsEpiSC lines will be needed to evaluate their future clinical potential.

The isolation of rsEpiSCs opens an interesting debate on the pluripotent state. How many different pluripotent states actually exist? How are the spatial differences produced, and do they first occur at the EpiSC stage or do they start even earlier? It is also unclear whether the different pluripotent stem cells represent fixed stages in embryonic development, or whether there is a gradual change in the epigenetic status of the cells in culture that enables the molecular and cellular characteristics of each of them. While more experimental validation are required, this study clearly demonstrates that a new type of pluripotent stem cell exists in mammals that may affect the way that we view developmental embryogenesis and regenerative medicine.

\section{Uri Weissbein ${ }^{1}$, Nissim Benvenisty $^{1}$ \\ ${ }^{I}$ The Azrieli Center for Stem Cells and Genetic Research, Department of Genetics, Silberman Institute of Life Sciences, The Hebrew Univer- sity, Jerusalem 91904, Israel \\ Correspondence: Nissim Benvenisty \\ E-mail: nissimb@mail.huji.ac.il}

\section{References}

1 Evans MJ, Kaufman MH. Nature 1981; 292:154-156.

2 Thomson JA, Itskovitz-Eldor J, Shapiro SS, et al. Science 1998; 282:1145-1147.

3 Ben-David U, Kopper O, Benvenisty N. Cell Stem Cell 2012; 10:666-677.

4 Brons IG, Smithers LE, Trotter MW, et al. Nature 2007; 448:191-195.

5 Tesar PJ, Chenoweth JG, Brook FA, et al. Nature 2007; 448:196-199.

6 Nichols J, Smith A. Cell Stem Cell 2009; 4:487-492.

7 Wu J, Okamura D, Li M, et al. Nature 2015; 521:316-321. 